\title{
Oxidative stress in pregnancy and reproduction
}

\section{Kate Duhig, Lucy C Chappell and Andrew H Shennan}

\begin{abstract}
Oxidative stress is implicated in the pathophysiology of many reproductive complications including infertility, miscarriage, pre-eclampsia, fetal growth restriction and preterm labour. The presence of excess reactive oxygen species can lead to cellular damage of deoxyribonucleic acids, lipids and proteins. Antioxidants protect cells from peroxidation reactions, limiting cellular damage and helping to maintain cellular membrane integrity. There is overwhelming evidence for oxidative stress causing harm in reproduction. However, there is sparse evidence that supplementation with commonly used antioxidants (mostly vitamins $\mathrm{C}$ and $\mathrm{E}$ ) makes any difference in overcoming oxidative stress or reversing disease processes. There may be potential for antioxidant therapy to ameliorate or prevent disease, but this requires a thorough understanding of the mechanism of action and specificity of currently used antioxidants.
\end{abstract}

\section{Keywords}

High-risk pregnancy, maternal-fetal medicine, oxidative stress, infertility, complications

Date received: I5 April 20I5; accepted: 13 April 2016

\section{Introduction}

Oxidative stress has been implicated in the pathophysiology of numerous diseases. Reactive oxygen species (ROS) are molecules containing contain at least one atom of oxygen and which have the potential to generate free radicals. At a physiological level, they are involved in cell signalling pathways, important to cellular function. ${ }^{1}$ However, the presence of excess ROS can lead to cellular damage of DNA, lipids and cellular proteins. ${ }^{2}$ Antioxidants protect cells from peroxidation reactions, limiting cellular damage and helping to maintain cellular membrane integrity. The term 'oxidative stress' is defined as an imbalance between pro- and antioxidant capacity. It is recognised to be a feature of acute and chronic diseases including cardiovascular, ${ }^{3}$ neurodegenerative ${ }^{4}$ and malignant disease..$^{5}$ Oxidative stress has been implicated in many reproductive and pregnancy disorders, from subfertility to miscarriage, maternal vascular disease and preterm labour. Antioxidant enzymes include glutathione peroxidase and superoxide dismutase, which are dependent on co-factors including selenium, zinc and iron. There may be potential for antioxidant therapy to ameliorate or prevent disease. Common antioxidants used in therapy include vitamins $\mathrm{A}, \mathrm{C}$ and $\mathrm{E}$, selenium, folic acid and flavonoids. This review will discuss the evidence for oxidative stress causing disease in human reproduction and the role of antioxidants.

\section{Fertility}

High levels of ROS in semen have been reported in men with reduced sperm motility. ${ }^{6}$ In vitro studies have supported the role for antioxidants in improving sperm motility. ${ }^{7}$ However, a recent systematic review of 48 randomised controlled trials revealed only four studies of 277 men, with low-quality evidence of an improvement in clinical pregnancy rates following antioxidant supplementation in men with subfertility (OR $4.21,95 \%$ CI 2.08 to $8.51, \mathrm{P}<0.0001){ }^{8}$

Oocyte quality is also affected by oxidative stress. ROS play an important role in cellular signalling for the induction of meiosis in the oocyte; yet conversely, high levels of ROS have been shown to impair oocyte maturation. ${ }^{9}$ Some evidence suggest that oocyte quality may be affected by an increase in ROS associated with advancing maternal age. Murine models have shown improved quantity and quality of oocytes with supplementation with vitamins $\mathrm{C}$ and $\mathrm{E}$, somewhat counteracting the effect of ageing. ${ }^{10}$ A small study of women undergoing IVF demonstrated a significant decrease in glutathione transferase and catalase, both associated with ROS scavenging capacity, in aspirates from ovarian follicular fluid from older women. ${ }^{11}$

Subfertility has been associated with cigarette smoking and alcohol consumption, in part thought to be mediated by an increase in ROS. ${ }^{12}$ A raised maternal body mass index has been shown to impact on female fertility, partially through menstrual irregularity and also increased spontaneous miscarriage rates have been documented. ${ }^{13}$ Normal pregnancy is associated with components of the metabolic syndrome: insulin resistance, hypertriglyceridemia, an up-regulation of the inflammatory cascade. Obese women experience an exaggeration of this metabolic adaptation and increased risk of pregnancy adverse outcome. ${ }^{14}$ Lipid peroxides are increased in normal pregnancy, ${ }^{15}$ but few findings are available for oxidative stress in obese pregnancy. Despite in vitro evidence to support a role for increased oxidative stress in impaired fertility, and some evidence for improved outcomes in animal models with antioxidants, a recent systematic review of randomised controlled trials comparing any type or dose of oral antioxidant use for women with subfertility found that their use was not associated with an increased clinical pregnancy or live birth rate (OR $1.25,95 \%$ CI 0.19 to $8.26, \mathrm{P}=0.82) .{ }^{16}$

\section{Miscarriage}

The majority of miscarriages are associated with genetic or chromosomal abnormalities in the developing embryo. Miscarriage affects approximately $25 \%$ of all pregnancies, the majority of which occur in the first trimester. Increased free radicals and ROS have been implicated in the pathophysiology of early pregnancy loss. There is evidence of rising mRNA and activity of antioxidant enzymes at the time when oxygen tension rises within the placental bed, during the period of normal placental differentiation. ${ }^{17}$ However, substantial increases in oxidative stress ${ }^{18}$ and loss of antioxidant defences ${ }^{19}$ are associated with

Women's Health Academic Centre, King's College London, London, UK

\section{Corresponding author:}

Andrew Shennan, Women's Health Academic Centre, King's College London, London SEI 7EH, UK.

Email: andrew.shennan@kcl.ac.uk 
early pregnancy loss. Serum prolidase, total antioxidant capacity and sulfhydryl levels (markers of oxidative stress) have been demonstrated to be significantly correlated in women with early pregnancy loss. ${ }^{20}$

Increased generation of ROS in blood granulocytes has been seen in women with recurrent miscarriages when compared to controls. ${ }^{21}$ Transgenic mice bred with mitochondrial respiratory chain dysfunction, and therefore increased oxidative stress, exhibit reduced fertility and increased miscarriage rates. ${ }^{22}$ Peroxiredoxins are a family of antioxidant proteins expressed by cytotrophoblastic cells. There is evidence to suggest that downregulation of these proteins is associated with miscarriage. $^{23}$

\section{Pre-eclampsia and fetal growth restriction}

Pre-eclampsia is a disorder associated with abnormal placentation and excessive maternal inflammatory vascular response. The mechanisms underpinning the development of pre-eclampsia are not fully understood, but dysfunction of vascular endothelium is thought to be central to the pathophysiology of the disease. Imperative to adequate placental function is the development of its vascular bed. In normal pregnancies, fetal cytotrophoblasts invade maternal spiral arterioles and enhance expansion of the vascular network. Pre-eclampsia is associated with abnormal trophoblastic invasion, leading to an alteration in this vascular remodelling and causing placental insufficiency. ${ }^{24}$ Some degree of hypoxia is thought to be essential in the normal placentation process, but defective trophoblastic invasion and remodelling of the uterine spiral arteries seen in pre-eclampsia is thought to result in excessive hypoxia at the placental bed. ${ }^{25}$ Pregnancies complicated by preeclampsia demonstrate increased indices of systemic oxidative stress when compared to normotensive counterparts. ${ }^{26}$

There is considerable scientific evidence to support oxidative stress in placental disease. Mitochondria are sources of ROS, and a small study has shown a $47 \%$ increase in mitochondrial protein in placental samples from pre-eclamptic pregnancies versus normotensive controls. ${ }^{27}$ Lipid peroxides are formed in the placenta due to membrane disruption by ROS and studies have shown significantly higher markers of lipid peroxidation in pre-eclampsia. ${ }^{28,29}$ Xanthine oxidase has been associated with reperfusion injury following periods of ischaemia via the generation of ROS and cytotrophoblasts from pre-eclamptic pregnancies have shown an increase in xanthine oxidase activity. ${ }^{30}$ The lumen of placental endoplasmic reticulum is the site responsible for protein synthesis and processing. Disturbance of normal homeostatic conditions, such as hypoxia induced oxidative stress, has been demonstrated to lead to an increase in luminal misfolded proteins, which can lead to cellular apoptosis. ${ }^{31}$ It is hypothesised that this pathway of apoptosis and protein misfolding at the placental interface may contribute to fetal growth restriction and promote the maternal preeclampsia syndrome via cytokine cascade and prostaglandin synthesis. ${ }^{2}$ In normal pregnancy, haemodynamic balance is determined by a number of circulating factors including prostaglandins, tipped in favour of vasodilatory prostaglandins (PGI2 and PGE). However, in women with preeclampsia, there is a relative increase in the vasoconstricting prostaglandins (thromboxane A2 and PGF2 alpha) such that the haemodynamic state often becomes vasoconstrictive. ${ }^{32}$ Isoprostanes are free-radical-catalysed prostaglandin isomers, considered to be reliable indicators of in vivo oxygen stress and lipid peroxidation, and are found to be raised in pre-eclampsia, reflective of this vasoconstrictive state. ${ }^{33}$

Uteroplacental insufficiency, due to reduced perfusion at spiral arterioles, is well recognised to cause fetal growth restriction. Markers of oxidative stress, including increased lipid peroxides ${ }^{34}$ and xanthine oxidase, ${ }^{35}$ have been seen in maternal serum, umbilical cord and placental tissue of pregnancies complicated with fetal growth restriction.

The weight of evidence of oxidative stress playing a key role in the development of pre-eclampsia led to the hypothesis that antioxidant supplementation might have a role in preventing the disease. A large, randomised placebo-controlled trial investigated supplementation of women at increased risk of developing pre-eclampsia with vitamins $\mathrm{C}$ and $\mathrm{E}$, and found no evidence that these antioxidants prevented the disorder. ${ }^{36}$ Evidence from a recent meta-analysis of over 6500 women supplemented with any oral antioxidant, for any duration did not support their use for the prevention of pre-eclampsia (RR $0.73,95 \%$ confidence intervals (CI) 0.51 to 1.06$).{ }^{37}$ In this analysis, antioxidants were found to be associated with an increased risk of antihypertensive therapy and antenatal admissions for hypertension, although this was not reflected in an increase in cases of pre-eclampsia. They were also found to be associated with an increased risk of preterm birth (vitamin C) in a small trial. It may be that antioxidant therapy has different effects in women with different risk factors, or that oxidative stress is relevant in the pathogenesis in only a subset of women. The women may already have had adequate concentrations of vitamins $\mathrm{C}$ and $\mathrm{E}$ before therapy $^{38}$ or the timing of the administration of antioxidants may be important. Burton and Jauniaux found that the initiation of intervillous blood flow at 8 to 10 weeks of gestation was associated with a burst of oxidative stress; it may be that antioxidants need to be present at the time of a relevant pro-oxidant challenge. ${ }^{39}$

\section{Preterm labour and preterm pre-labour rupture of membranes}

The underlying mechanisms leading to spontaneous preterm labour are not fully understood. Evidence for oxidative stress in women with cervical incompetence is inconsistent, ${ }^{40}$ and this may reflect the heterogeneous nature of this population. Another study has reported significantly decreased serum selenium concentrations in women who went on to deliver preterm compared to controls. ${ }^{41}$

At term, oxidative stress is associated with placental membrane disruption and is thought to contribute to the onset of labour. ${ }^{42}$ It is thought that oxidative stress may be in part responsible for some cases of preterm pre-labour rupture of membranes (PPROM), possibly mediated through infection. Degradation of collagens within the chorioamnion may cause a defect and rupture in the fetal membranes causing a leak of amniotic fluid. Bacteria may play an important role in this process, with generation of ROS from immune cells as they kill bacteria, thought to damage amnion. ${ }^{43}$ Matrix metalloproteinase activity, a marker of oxidative stress, has been shown to be increased in human fetal membranes exposed to superoxide anions (a by-product of macrophages and neutrophils). ${ }^{44}$ Fetal telomere length (a marker of cellular biological age and measured as a surrogate for oxidative stress) from cord blood leucocytes is decreased in PPROM compared to preterm labour with intact membranes, suggesting acceleration of cellular ageing in the PPROM group via oxidative stress. ${ }^{45}$ Analysis of amniotic fluid samples collected by transabdominal amniocentesis in women with PPROM demonstrates decreased total antioxidant capacity, but no difference in markers of oxidative stress regardless of whether microbial invasion or frank chorioamnionitis was present or not. ${ }^{46}$ In vitro studies of chorioamnion membranes exposed to antioxidants (vitamins C and E) resist ROS-induced tissue damage. ${ }^{47}$ In spite of this theoretical evidence, secondary analyses have not confirmed that supplementation with vitamins $\mathrm{C}$ and $\mathrm{E}$ is protective in women with PPROM. $^{48}$

Analysis of ROS and total antioxidant potential in cord blood from preterm infants demonstrate that preterm infants have significantly decreased antioxidant potential when compared to term counterparts. ${ }^{49}$ This lack of antioxidant capacity leaves preterm neonates susceptible to ROS-induced cellular damage with associated bronchopulmonary dysplasia and retinopathy. ${ }^{50}$

\section{Conclusion and future directions}

This review has summarised the evidence for oxidative stress causing harm in reproduction. However, the paradox remains that therapy with antioxidants in a range of disorders has been disappointing or 
even harmful, and the explanation for this remains elusive. Some early trials demonstrated that antioxidant therapy reversed markers of oxidative stress, ${ }^{51}$ but subsequent large trials have shown no effect on clinical outcomes. Oxidative stress may be a bystander phenomenon, rather than fundamental to the aetiology of some of these diseases. Other explanations may relate to unintended effects of antioxidant supplementation; for example, vitamin $\mathrm{E}$ has many isoforms, and therapeutic preparations of alpha tocopherol result in competitive inhibition of gamma tocopherol, itself associated with anti-inflammatory properties. ${ }^{2}$

Antioxidant enzymes include glutathione peroxidase and superoxide dismutase, which are dependent on co-factors including selenium, zinc and iron. It may be naïve to assume that the mechanism of action and specificity of currently used antioxidants is correct for all diseases associated with oxidative stress. The negative findings of therapeutic trials may discourage research in this area, but scientific mechanisms of disease are needed, particularly mechanism of antioxidant therapy, before further large-scale trials of antioxidant therapy are funded. Other trials investigating prophylactic agents to prevent pre-eclampsia are ongoing. For example, the FACT trial (high-dose folic acid supplementation throughout pregnancy for the pre-eclampsia prevention), a randomised, double-blind placebo-controlled efficacy study has recently finished recruiting and final data collection is ongoing. Well-designed basic science and clinical trials need to be pursued together if we are to impact on outcomes in pregnancy and reproduction.

\section{Declaration of conflicting interests}

The author(s) declared no potential conflicts of interest with respect to the research, authorship, and/or publication of this article.

\section{Funding}

The author(s) received no financial support for the research, authorship, and/or publication of this article.

\section{Guarantor}

AS.

\section{Contributorship}

KD made a substantial contribution to the acquisition of the evidence, drafted the article, revised it critically for important intellectual content and approved the version to be published. LC also drafted the article, revised it critically for important intellectual content and approved the version to be published. AS was involved in drafting the article, revising it critically for important intellectual content and approving the version to be published.

\section{References}

1. Pereira RD, De Long NE, Wang RC, et al. Angiogenesis in the placenta: the role of reactive oxygen species signaling. BioMed Res Int 2015; 2015: 814543.

2. Poston L, Igosheva N, Mistry HD, et al. Role of oxidative stress and antioxidant supplementation in pregnancy disorders. Am J Clin Nutr 2011; 94(6 Suppl): 1980s-1985s.

3. Dhalla NS, Temsah RM and Netticadan T. Role of oxidative stress in cardiovascular diseases. J Hypertension 2000; 18: 655-673.

4. Lin MT and Beal MF. Mitochondrial dysfunction and oxidative stress in neurodegenerative diseases. Nature 2006; 443: 787-795.

5. Khansari N, Shakiba Y and Mahmoudi M. Chronic inflammation and oxidative stress as a major cause of age-related diseases and cancer. Recent Pat Inflamma Allergy Drug Discovery 2009; 3: 73-80.

6. Agarwal A and Saleh RA. Role of oxidants in male infertility: rationale, significance, and treatment. Urol Clin North Am 2002; 29: $817-827$.
7. Baker HW, Brindle J, Irvine DS, et al. Protective effect of antioxidants on the impairment of sperm motility by activated polymorphonuclear leukocytes. Fertil Steril 1996; 65: 411-419.

8. Showell MG, Brown J, Yazdani A, et al. Antioxidants for male subfertility. Cochrane Database Syst Rev 2011; 1: Cd007411.

9. Behrman HR, Kodaman PH, Preston SL, et al. Oxidative stress and the ovary. J Soc Gynecol Invest 2001; 8(1 Suppl Proceedings): S40-S42.

10. Tarin JJ, Perez-Albala S and Cano A. Oral antioxidants counteract the negative effects of female aging on oocyte quantity and quality in the mouse. Mol Reprod Dev 2002; 61: 385-397.

11. Carbone MC, Tatone C, Monache DS, et al. Antioxidant enzymatic defences in human follicular fluid: characterization and agedependent changes. Mol Hum Reprod 2003; 9: 639-643.

12. Ruder EH, Hartman TJ, Blumberg J, et al. Oxidative stress and antioxidants: exposure and impact on female fertility. Hum Reprod Update 2008; 14: 345-357.

13. Pasquali R, Patton L and Gambineri A. Obesity and infertility. Curr Opin Endocrinol Diabetes Obes 2007; 14: 482-487.

14. Jarvie E, Hauguel-de-Mouzon S, Nelson SM, et al. Lipotoxicity in obese pregnancy and its potential role in adverse pregnancy outcome and obesity in the offspring. Clin Sci 2010; 119: 123-129.

15. Little RE and Gladen BC. Levels of lipid peroxides in uncomplicated pregnancy: a review of the literature. Reprod Toxicol 1999; 13: $347-352$.

16. Showell MG, Brown J, Clarke J, et al. Antioxidants for female subfertility. Cochrane Database Syst Rev 2013; 8: Cd007807.

17. Jauniaux E, Watson AL, Hempstock J, et al. Onset of maternal arterial blood flow and placental oxidative stress. A possible factor in human early pregnancy failure. Am J Pathol 2000; 157: 2111-2122.

18. Vural P, Akgul C, Yildirim A, et al. Antioxidant defence in recurrent abortion. Clin Chim Acta 2000; 295: 169-177.

19. Simsek M, Naziroglu M, Simsek H, et al. Blood plasma levels of lipoperoxides, glutathione peroxidase, beta carotene, vitamin A and $\mathrm{E}$ in women with habitual abortion. Cell Biochem Funct 1998; 16: 227-231.

20. Toy H, Camuzcuoglu H, Camuzcuoglu A, et al. Decreased serum prolidase activity and increased oxidative stress in early pregnancy loss. Gynecol Obstet Invest 2010; 69: 122-127.

21. Safronova VG, Matveeva NK, Avkhacheva NV, et al. Changes in regulation of oxidase activity of peripheral blood granulocytes in women with habitual abortions. Bull Exp Biol Med 2003; 136: 257-260.

22. Ishii T, Miyazawa M, Takanashi $\mathrm{Y}$, et al. Genetically induced oxidative stress in mice causes thrombocytosis, splenomegaly and placental angiodysplasia that leads to recurrent abortion. Redox Biol 2014; 2: 679-685.

23. Gharesi-Fard B. Preoxiredoxin family members (Prx3 and Prx4) and pregnancy disorder (recurrent pregnancy loss). Meth Mol Biol 2015; 1208: 299-311.

24. Lim KH, Zhou Y, Janatpour M, et al. Human cytotrophoblast differentiation/invasion is abnormal in pre-eclampsia. Am J Pathol 1997; 151: 1809-1818.

25. Redman CW and Sargent IL. Placental debris, oxidative stress and pre-eclampsia. Placenta 2000; 21: 597-602.

26. McKinney ET, Shouri R, Hunt RS, et al. Plasma, urinary, and salivary 8-epi-prostaglandin f2alpha levels in normotensive and preeclamptic pregnancies. Am J Obstet Gynecol 2000; 183: 874-877.

27. Wang Y and Walsh SW. Placental mitochondria as a source of oxidative stress in pre-eclampsia. Placenta 1998; 19: 581-586.

28. Wu JJ. Lipid peroxidation in preeclamptic and eclamptic pregnancies. Eur J Obstet Gynecol Reprod Biol 1996; 64: 51-54.

29. Patil SB, Kodliwadmath MV and Kodliwadmath SM. Lipid peroxidation and antioxidant status in hypertensive pregnancies. Clin Exp Obstet Gynecol 2008; 35: 272-274. 
30. Many A, Hubel CA, Fisher SJ, et al. Invasive cytotrophoblasts manifest evidence of oxidative stress in preeclampsia. Am J Pathol 2000; 156: 321-331.

31. Burton GJ, Yung HW, Cindrova-Davies T, et al. Placental endoplasmic reticulum stress and oxidative stress in the pathophysiology of unexplained intrauterine growth restriction and early onset preeclampsia. Placenta 2009; 30(Suppl A): S43-S48.

32. Friedman SA. Preeclampsia: a review of the role of prostaglandins. Obstet Gynecol 1988; 71: 122-137.

33. Turpin CA, Sakyi SA, Owiredu WK, et al. Association between adverse pregnancy outcome and imbalance in angiogenic regulators and oxidative stress biomarkers in gestational hypertension and preeclampsia. BMC Pregnancy Childbirth 2015; 15: 189.

34. Karowicz-Bilinska A. Lipid peroxides concentration in women with intrauterine growth restriction. Ginekologia Polska 2004; 75: 6-9.

35. Biri A, Bozkurt N, Turp A, et al. Role of oxidative stress in intrauterine growth restriction. Gynecol Obstet Invest 2007; 64: 187-192.

36. Poston L, Briley AL, Seed PT, et al. Vitamin C and vitamin E in pregnant women at risk for pre-eclampsia (VIP trial): randomised placebo-controlled trial. Lancet 2006; 367: 1145-1154.

37. Rumbold A, Duley L, Crowther CA, et al. Antioxidants for preventing pre-eclampsia. Cochrane Database Syst Rev 2008; 1: Cd004227.

38. Padayatty SJ and Levine M. Vitamins $\mathrm{C}$ and $\mathrm{E}$ and the prevention of preeclampsia. $N$ Engl J Med 2006; 355: 1065; author reply 6.

39. Burton GJ and Jauniaux E. Placental oxidative stress: from miscarriage to preeclampsia. J Soc Gynecol Invest 2004; 11: 342-352.

40. Zolotukhin P, Aleksandrova A, Goncharova A, et al. Oxidative status shifts in uterine cervical incompetence patients. Syst Biol Reprod Med 2014; 60: 98-104.

41. Rayman MP, Wijnen H, Vader H, et al. Maternal selenium status during early gestation and risk for preterm birth. CMAJ 2011; 183: $549-555$.
42. Agarwal A, Gupta S and Sharma RK. Role of oxidative stress in female reproduction. Reprod Biol Endocrinol 2005; 3: 28.

43. Woods JR Jr. Reactive oxygen species and preterm premature rupture of membranes-a review. Placenta 2001; 22(Suppl A): S38-S44.

44. Buhimschi IA, Kramer WB, Buhimschi CS, et al. Reduction-oxidation (redox) state regulation of matrix metalloproteinase activity in human fetal membranes. Am J Obstet Gynecol 2000; 182: 458-464.

45. Menon R, Yu J, Basanta-Henry P, et al. Short fetal leukocyte telomere length and preterm prelabor rupture of the membranes. PloS One 2012; 7: e31136.

46. Kacerovsky M, Tothova L, Menon R, et al. Amniotic fluid markers of oxidative stress in pregnancies complicated by preterm prelabor rupture of membranes. J Matern Fetal Neonatal Med 2014; 27: 1-10.

47. Plessinger MA, Woods JR Jr and Miller RK. Pretreatment of human amnion-chorion with vitamins $\mathrm{C}$ and $\mathrm{E}$ prevents hypochlorous acid-induced damage. Am J Obstet Gynecol 2000; 183: 979-985.

48. Spinnato JA 2nd, Freire S, Pinto e Silva JL, et al. Antioxidant supplementation and premature rupture of the membranes: a planned secondary analysis. Am J Obstet Gynecol 2008; 199: 433.e1-8.

49. Georgeson GD, Szony BJ, Streitman K, et al. Antioxidant enzyme activities are decreased in preterm infants and in neonates born via caesarean section. Eur J Obstet Gynecol Reprod Biol 2002; 103: 136-139.

50. Auten RL and Davis JM. Oxygen toxicity and reactive oxygen species: the devil is in the details. Pediatr Res 2009; 66: 121-127.

51. Chappell LC, Seed PT, Kelly FJ, et al. Vitamin C and E supplementation in women at risk of preeclampsia is associated with changes in indices of oxidative stress and placental function. Am J Obstet Gynecol 2002; 187: 777-784. 